\title{
Alocação escalonar ou seqüencial x Alocação recíproca ou matricial: uma análise comparativa
}

\author{
Staggered or sequential allocation $x$ reciprocal or matrix allocation: a \\ comparative analysis
}

\author{
Paulo César Souza \\ Doutorando em Ciências da Saúde (UFMT) \\ Professor da Universidade do Estado de \\ Mato Grosso (UNEMAT) \\ Contador da Secretaria de Estado de Saúde \\ (SES-MT) \\ João Henrique Gurtler Scatena \\ Ph.D em Medicina Preventiva (USP) \\ Doutor em Saúde Pública (FSP/USP) \\ Professor da Universidade Federal de Mato \\ Grosso (UFMT)
}

\author{
Leandro da Silva Medeiros \\ Bacharel em Ciências da Computação \\ (UNEMAT)
}

\section{Resumo}

Uma das questões que é motivo de debates no que se refere à apuração dos custos se refere ao rateio dos custos indiretos. Para minimizar as distorções e as arbitrariedades surgiu o custeio por departamentos ou centros de custos. Ainda assim, permanece uma questão: qual metodologia usar para distribuir os custos dos centros administrativos e intermediários aos finais e externos? Existem basicamente três metodologias que são aplicáveis a vários ramos de atividades, em especial aos hospitais: alocação direta, alocação escalonar ou sequencial e alocação recíproca ou matricial. Este trabalho é oriundo de um estudo de caso realizado num hospital de médio porte do interior do Estado de Mato Grosso e objetiva comparar os valores obtidos através da aplicação das três metodologias, com destaque para as duas últimas. $\mathrm{O}$ resultado demonstrou que no hospital em estudo, a diferença dos valores apurados pela alocação recíproca em relação à escalonar ou sequencial não foi tão expressiva, no entanto, ratificou a afirmação dos autores de que a alocação recíproca proporciona valores mais precisos. Ficou demostrado também que a diferença nos valores das duas metodologias poderia ser maior, caso houvesse uma maior reciprocidade na prestação de serviços entre os centros administrativos e intermediários.

Palavras-chave: Gestão de custos; Contabilidade de custos; Custos hospitalares.

\begin{abstract}
:
One of the issues is a matter of debate in relation to costs assessment refers to the apportionment of overheads. To minimize distortion and arbitrary emerged costing of departments or cost centers. Still, one question remains: what methodology to use to distribute the costs of administrative centers and intermediaries to end users and external? There are basically three approaches that are applicable to many branches of activities, especially to hospitals: direct allocation, allocation and assignment schedule or sequential or reciprocal matrix. This work is from a case study in a medium-sized hospital in the state of Mato Grosso and objectively compare the values obtained by applying the three methods, especially the last two. The result showed that the hospital under study, the difference in values determined by allocating each other in relation to the scale or sequence was less pronounced, however, ratified the authors' assertion that the reciprocal allocation provides more precise values. Also been demonstrated that the difference in values of both
\end{abstract}


methodologies could be higher if there was more reciprocity in providing services between the administrative centers and intermediaries.

Key-Words: Cost management. Cost Accounting. Hospital costs.

\section{INTRODUÇÃO}

No processo de apuração de custos existem algumas tarefas que são simples de serem realizadas, estando livres de dilemas, dúvidas e críticas e outras bastante complexas e dotadas de dúvidas, dilemas e opções possíveis. A apuração dos custos diretos é uma das tarefas mais simples e objetivas. No entanto, o processo de distribuição, ou seja, rateio dos custos indiretos está repleto de dilemas e dúvidas, de modo que as escolhas podem influenciar os valores dos custos finais. A grande crítica feita aos métodos de custeio que realizam o rateio dos custos indiretos é o alto grau de arbitrariedade que influencia no valor final de custos, de modo que, a escolha por uma ou outra forma de rateio pode tornar um produto lucrativo em deficitário ou vice-versa.

Diante dessa grande questão há muito existente no âmbito dos estudos relacionados aos custos, surgiu o custeio por departamentos ou centro de custos, o qual, segundo Bruni e Fama (2004, p. 129) tem como uma das vantagens a "melhor identificação e alocação dos custos indiretos de fabricação". Embora essa forma de distribuição dos custos indiretos torne o processo mais racional, não é totalmente livre de dilemas. Um dos principais, está na escolha da metodologia de alocação dos custos entre os departamentos ou centros de custos de produção e de serviços. Existem três metodologias utilizadas, indo da mais simples à mais complexa, as quais são: alocação direta, alocação escalonar ou sequencial e alocação recíproca ou matricial. Assim, os totais dos centros de custos produtivos e consequentemente dos valores unitários poderão sofrer alterações dependendo da metodologia adotada.

Segundo Martins (1996), a segunda é a mais utilizada embora apresente imperfeições e a última só é possível de operacionalizar mediante adoção de recursos eletrônicos para processamento de dados em virtude do grande volume de cálculos a efetuar, embora seja mais precisa.

Diante dessas duas metodologias, a grande pergunta é: Comparando-se os valores de custos, mediante adoção das duas últimas metodologias, verificar-se-à diferenças significativas? Este trabalho objetiva, realizar esta comparação, com base num estudo de caso realizado num hospital público de médio porte localizado no médio-norte do Estado de Mato Grosso.

\section{REFERENCIAL TEÓRICO}

\subsection{Custeio por departamentos ou centro de custos}

Um dos primeiros passos, ao se iniciar um processo de apuração de custos em hospitais, é definir qual é o objeto de custo que se quer mensurar e qual método de custeio será adotado.

Objeto de custo é definido por Ching (2001, p. 20) como "qualquer coisa que se deseja medir o custo ou que necessite de uma medida de custo separado". Pode-se desejar conhecer o custo por especialidade, departamento ou centro de custos, paciente-dia, exame, atendimento, procedimento, etc.

Para Padoveze (2006, p.150), método de custeio é definido como "o processo de identificar o custo unitário de um produto ou serviço ou de todos os produtos e serviços de uma empresa, partindo do total dos custos diretos e indiretos". Os principais métodos de custeio são: teoria das restrições, custeio por absorção, custeio $\mathrm{ABC}$, custeio RKW, custeio direto ou variável. 
No processo de apuração de custos hospitalares, a experiência tem mostrado que a divisão da organização em centros de custos traz bons resultados, pois, ao invés de lidar com a organização como um todo, segrega-se a mesma em centros de custos ou departamentos, facilitando o processo de obtenção das informações e acompanhamento das atividades.

Segundo Bruni e Famá (2004), a utilização da departamentalização ou centro de custos traz como vantagens a melhoria dos controles internos e a redução dos problemas de relacionados ao rateio dos custos indiretos.

O processo de classificação dos centros de custos e elaboração do plano de custos é a primeira etapa no desenvolvimento de um sistema de apuração de custos hospitalares, após a definição do método de custeio e do objeto de custo. Para isso, geralmente, se faz necessário a realização de visitas à organização com o objetivo de conhecer o processo de trabalho e o fluxo das informações.

Mas, pergunta-se: o que é um centro de custos? Como definir um centro de custos? Quais os critérios para determinar um espaço ou setor como um centro de custos?

Para Martins (1996), um centro de custo pode ser um departamento da organização, assim como, um departamento pode ser subdividido em dois ou mais centros de custos. Para esse autor, um departamento é a unidade mínima administrativa para a contabilidade de custos, representada por homens e máquinas, que desenvolve atividades homogêneas.

$\mathrm{Na}$ visão de Falk (2001, p. 33), o centro de custos é chamado também de centro de responsabilidade, sendo definido como "uma unidade organizacional do hospital para qual existe chefia com autoridade de mando e com responsabilidade perante os resultados alcançados".

Segundo Beulke e Bertó (2008, p. 32), um "centro de custos é uma unidade operacional autônoma, distinta das demais, "pulsando" em conseqüência, uma vida própria (...)".

De acordo com o Ministério da Saúde (MS, 2006, p. 12), um centro de custos é um "setor de uma instituição que possui gastos mensuráveis". O Ministério da Saúde destaca ainda que um centro de custos, não tem que acompanhar necessariamente o organograma da instituição, sendo necessário, às vezes, dividir um setor em mais de um centro de custos para facilitar o processo de apuração e alocação dos custos.

Para a classificação de um setor ou atividade como um centro de custos deve-se observar, segundo Falk (2008, p. 79), os seguintes critérios:

a) ocupação de espaço físico próprio;

b) alocação de mão-de-obra própria;

c) consumo de material numa atividade exclusiva.

Se o setor que se pretende classificar como centro de custos não atende a esses critérios é preferível subordiná-lo a outro centro de custos dentro de seu segmento.

Os centros de custos podem ser classificados sob diversas categorias dependendo do grau de detalhamento que a instituição deseja em termos de informações, sendo possível a classificação dos centros de custos em dois ou mais tipos conforme se apresenta a seguir.

Apresentando uma classificação generalista aplicada a todos os ramos de atividade, Bruni e Famá (2004) classificam os departamentos ou centros de custos em dois grandes grupos: de serviços e de produção.

Uma classificação simplista para os hospitais é proposta por Falk (2001, p. 48), dividindo os centros de custos em dois tipos:

Centros de custos produtores de renda: são "os centros de custos onde o consumo de recursos pode ser designado facilmente aos pacientes, individualmente". Ex: ambulatório, pronto-socorro, unidades de internação, etc. 
Centros de custos de apoio: são os "centros de custos que prestam serviços que não podem ser relacionados com um paciente específico". Ex: lavanderia, manutenção, transportes, faturamento, etc.

Para o autor citado acima, o primeiro tipo poderia ser chamado de centro de receita, pois são eles que geram as receitas para a instituição. $O$ segundo tipo poderia ser chamado apenas de centro de custos, pois os mesmos apenas absorvem custos, mas não geram receitas diretamente.

Avançando no grau de detalhamento da classificação por tipos, o Ministério da Saúde (MS, 2006, p. 20) divide os centros de custos em quatro tipos: Centro de custos administrativos, centro de custos intermediários, centro de custos final (produtivo) e centro de custos externos. Destaca-se que, o centro de custos externos é o "centro de custos que recebe os custos das atividades ou serviços prestados a pacientes não vinculados ao hospital ou a outras instituições (demanda externa)".

Existem ainda outras opções que apresentam maior grau de detalhamento como, por exemplo, a classificação utilizada pelo Hospital Universitário de Porto Alegre apresentada por Ching (2001, p. 42): centro de custos administrativos, centro de custos de base, centro de custos auxiliares ou intermediários, Centro de custos finais e Centro de custos de produção.

Como se pode notar, a classificação dos centros de custos por tipo pode ser realizada de várias formas com maior ou menor nível de detalhamento e também em conformidade com o tamanho e a complexidade da organização. Mediante essa classificação, torna-se possível elaborar o plano de centro de custos e, através dele, atribui-se um código a cada centro de custos para facilitar sua identificação e a alocação dos custos através de sistema informatizado. Após a classificação dos centros de custos, o próximo passo no processo de estruturação de um sistema de apuração de custos hospitalares é a escolha da metodologia de alocação desses custos.

\subsection{Metodologias de alocação dos custos indiretos}

A fim de tornar mais equânime a distribuição dos custos, de modo a se obter os custos por unidade de serviço produzido, surgiu o chamado custeio por departamentos ou centro de custos. Nesse sistema de acumulação, a organização é dividida em centro de custos, os custos diretos são alocados diretamente aos centros de serviço e aos produtivos, os custos indiretos gerais são distribuídos a todos os centros, os custos dos centros de serviço são distribuídos aos centros finais ou produtivos com base em critérios diversos de rateio e os custos totais acumulados nos centros produtivos são divididos pela quantidade de serviços produzidos, obtendo-se assim o valor de cada unidade de serviço produzido.

Ao tratar sobre as metodologias de alocação dos custos indiretos, Araújo e Nascimento (2007, p. 02) ressaltam que para o processo de mensuração de custos ser bem sucedido, uma atenção especial deve ser dada aos custos gerados pelos departamentos de suporte (centro de custos) e à maneira como estes são distribuídos entre si e aos departamentos de produção (centro de custos)".

Antes de prosseguir discorrendo sobre as metodologias de alocação, é de suma importância definir o que é alocação de custos. Segundo Falk (2001) a expressão alocação de custos ou alocação de despesas é o "[...] ato de levar (ratear) gastos ocorridos e registrados em alguma área ou objeto de custos para outras áreas."

Seguindo esta linha de pensamento HRQ Consultoria [200-], define alocação de custos como sendo:

[...] uma metodologia para o rateio dos custos indiretos da empresa, alocando-os para os objetos de custos que podem ser produtos, serviços, projetos, fases, centro de custos, unidade de negocio, família de produto ou qualquer dimensão que a empresa precise para alocar e controlar os seus custos diretos e indiretos. 
Para Araujo e Nascimento (2007), na maioria das organizações existe uma estrutura departamental constituída de diversos setores de suporte e vários setores de produção ou final. A alocação de custo vem para auxiliar na apropriação aos setores ou centros, dos custos gerados nas atividades executadas pelos setores de apoio e administrativos, e dos produtivos para os produtos.

Segundo Falk (2001), existem dois tipos de alocação de custos. A primeira seria alocar os custos indiretos dentro de um mesmo centro de custo, a fim de conhecer o custo total de algum produto ou serviço. Outro tipo seria alocar os custos totais de um centro de custos qualquer para outro centro que recebe serviços prestados por ele, e, assim, até chegarem aos centros finais e daí ratear para os produtos ou serviços, dependendo da metodologia de custo utilizada. $\mathrm{O}$ autor cita que existem três metodologias de alocação de custos conhecidas: alocação direta, alocação sequêncial ou escalonar, e alocação recíproca ou matricial.

\subsubsection{Alocação Direta}

Segundo Falk (2001, p. 61) essa metodologia de alocação é a mais simples das existentes. $\mathrm{O}$ autor ainda cita que "[...] todas as despesas de cada centro de custo de apoio são rateadas diretamente aos centros de custos produtores de renda." Em outras palavras, os gastos são rateados dos centros de apoio aos centros produtores, um a um, até terminar todos eles.

Conforme Souza (2009) esse método de alocação não considera a troca de serviços entre os centros de custos de apoio e administrativo, considerando que, de qualquer forma, no fim todos os custos serão transferidos para os centros produtores.

A principal vantagem dessa metodologia é a simplicidade para a sua operacionalização, no entanto, vale ressaltar que algumas distorções poderão ocorrer, sobrecarregando mais um centro produtivo do que outro por não levar em consideração o relacionamento entre os centros de custos de apoio ou de serviço. A Figura 1 demonstra o funcionamento desse método de alocação.

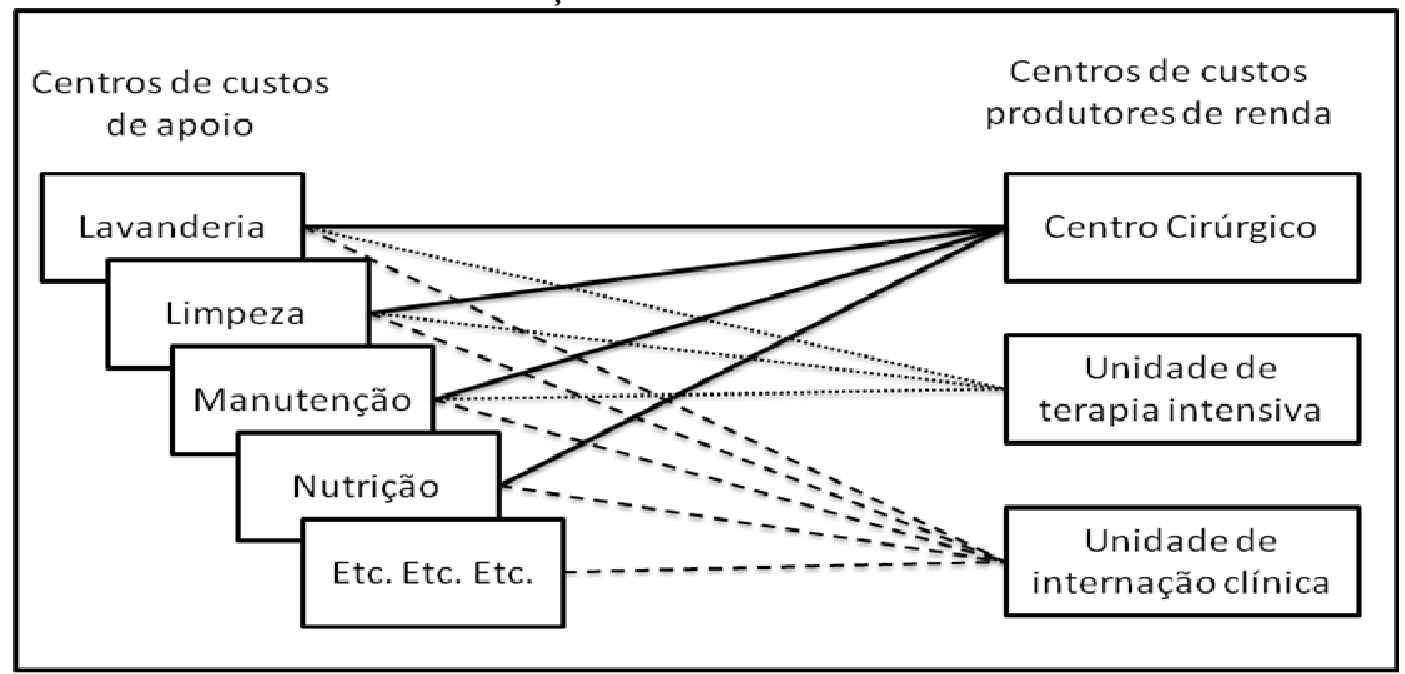

Figura 1 - Alocação Direta dos centros de custos de apoio. Fonte: Falk (2001)

\subsubsection{Alocação escalonar ou sequencial}

Esse método reconhece que existe uma troca de serviços entre os centros de custos de apoio. Inicialmente, é necessário definir o primeiro centro de apoio a ter os custos rateados e a ordem dos demais centros de custos (FALK, 2001).

Segundo Falk (2001) para realizar a ordenação dos centros de custos, é necessário utilizar algum critério. $\mathrm{O}$ autor cita que este critério pode ser pela quantidade de prestação de 
serviço que um centro realiza aos demais da instituição, ou pela quantidade de custo absorvida por cada centro, ambos da maior para a menor.

Comentando sobre as vantagens de utilizar esse método de alocação na apuração de custos, Ministério da Saúde (2006) cita que a principal vantagem desse método é a possibilidade de se apurar o total dos custos dos centros de apoio, bem como sua composição considerando o valor que o centro já possuía mais o que ele recebeu do centro que estava localizado acima dele na ordem de rateio. Uma ressalva sobre esse método é feita por Souza $(2009$, p. 74) quando afirma que "esse método ainda está sujeito a distorções, pois os valores alocados podem variar dependendo da seqüencia de alocação escolhida." Levando em consideração essa afirmação, ao definir a ordem de alocação dos centros de custos, exige-se uma atenção redobrada quanto ao critério de ordenação.

Na visão de Falk (2001), esse método não reconhece a reciprocidade entre os centros de custos de apoio, sendo a alocação de despesas sempre unidirecional. O autor ainda afirma que mesmo reconhecendo a troca de serviços entre os centros de custos de apoio, o método apresenta certo grau de distorção da realidade por desprezar a reciprocidade. A Figura 22 ilustra o processo de alocação sequencial.

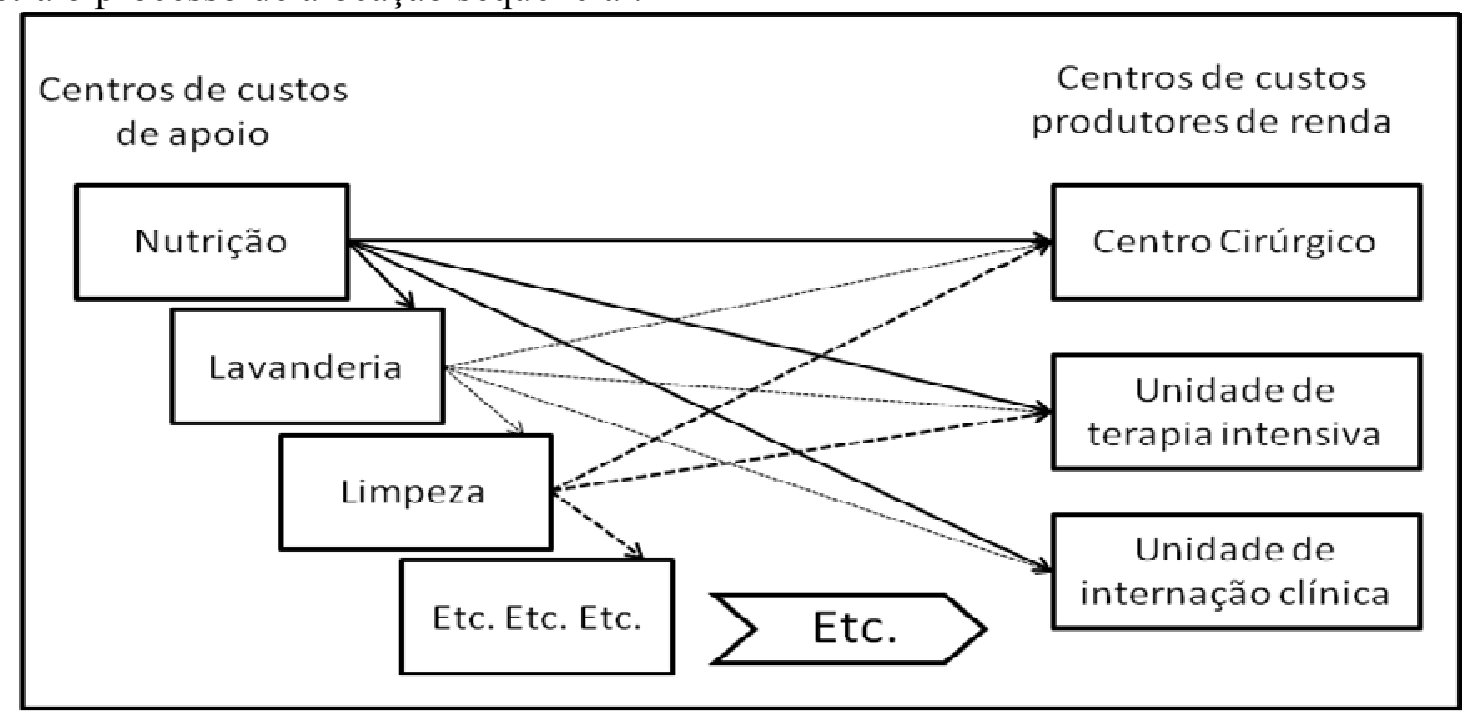

Figura 2 - Alocação Escalonar dos custos indiretos. Fonte: Falk (2001)

\subsubsection{Alocação recíproca ou matricial}

Segundo o Ministério da Saúde (2006, p. 27), a alocação recíproca é conhecida também como alocação matricial. $\mathrm{O}$ autor cita que "este método reconhece a reciprocidade entre todos os centros de custos não-produtivos, por isso apresenta melhores resultados." Porém, esse método é mais complexo que os demais mencionados anteriormente.

De acordo com Falk (2001), essa metodologia tenta eliminar as distorções relatadas anteriormente utilizando funções algébricas, desprezando assim a utilização de uma sequencia de rateio dos centros de custos de apoio. A Figura3 demonstra como se dá o processo de alocação recíproca.

Segundo Falk (2008), esse método é difícil de ser entendido e mais difícil ainda de ser implementado por se utilizar de álgebra matricial para a realização dos cálculos, sendo sua utilização ainda limitada mesmo nos Estados Unidos. No entanto, com a utilização dos recursos computacionais, para a realização dos cálculos, o método pode ser aplicado com mais facilidade.

Segundo Scardoelli et.al. (2003, p. 03), “além de tratar-se de um método complexo de alocação de custos, o método recíproco também exige certa dose de cálculos matemáticos, principalmente no tocante a álgebra matricial, envolvendo soma, multiplicação, inversão e 
transposição matricial”. No entanto, a aplicação desse método é facilitada se operacionalizada através de um software específico de apuração de custos.

A existência de software tem ajudado muito na utilização de metodologias mais complexas, mas operacionais em virtude de não requerer que os usuários entendam de álgebra matricial para trabalhar com o software. Mesmo assim, a utilização dessa metodologia ainda é bastante limita (FALK, 2001).

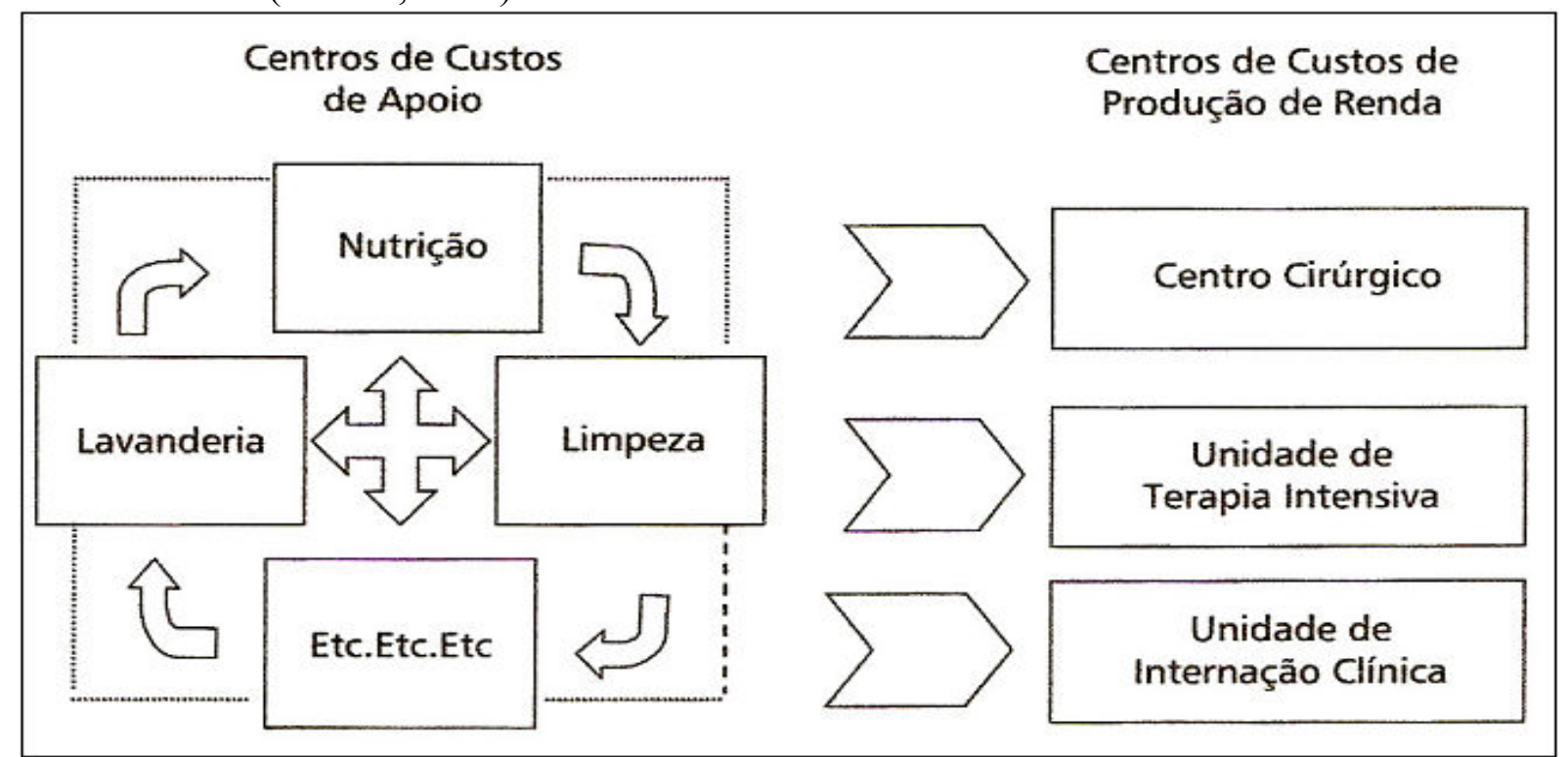

FIGURA 3 - Alocação Recíproca dos custos indiretos. Fonte: Falk (2001)

\section{METODOLOGIA}

Este trabalho é oriundo de um estudo de caso realizado no Hospital Municipal de Barra do Bugres, Mato Grosso, o qual é uma instituição pública, mantida com recursos municipais, conveniado com a Secretaria Estadual de Saúde de Mato Grosso e vinculado ao Sistema Único de Saúde.

De acordo com o Cadastro Nacional de Estabelecimentos de Saúde (CNES, 2008), o hospital tem sua estrutura composta por 80 leitos, sendo estes divididos entre as clínicas médica, cirúrgica, obstétrica e pediátrica.

A pesquisa realizada é do tipo "Estudo de Caso", pois segundo Martins (1994,), no estudo de caso o pesquisador dedica-se a estudos intensivos do passado, presente e de interações ambientais de uma (ou de algumas) unidade social: indivíduo, grupo, instituição, comunidade.

A abordagem foi de natureza qualitativa em relação ao processo de análise da instituição e definição da forma de rateio dos custos indiretos. A posição do pesquisador foi chamada de "observador participante", de acordo com a classificação proposta por Kider apud Martins (1994). Nesse tipo de pesquisa o observador é parte do contexto que está sendo observado, de forma que ele modifica e é modificado por esse contexto.

Durante a realização de uma pesquisa do curso de Mestrado em Saúde Coletiva pela Universidade Federal de Mato Grosso foi desenvolvido e implantado um software básico para o levantamento de algumas informações necessárias ao processo de apuração de custos do hospital.

Esse software foi desenvolvido por um bolsista do curso de ciências da computação da Universidade do Estado de Mato Grosso (UNEMAT) e foi denominado Sistema Integrado de Gestão Hospitalar (SIGHO), informatizando e integrando alguns setores do hospital por meio dos módulos do sistema e, também, um módulo específico para apuração de custos. O referido sistema foi implantado e realizada a apuração dos custos do hospital no período compreendido 
entre 15/10/2008 a 14/11/2008. Na tecnologia de desenvolvimento, foi utilizado o compilador Delphi 7 juntamente com a linguagem de programação "Object Pascal". Quanto ao banco de dados, foi utilizado o "Firebird 2.1", um poderoso Sistema de Gerenciamento de Banco de Dados (SGBD), baseado na tecnologia cliente/servidor.

O módulo destinado ao processamento dos dados para a apuração de custos foi modelado de modo a utilizar a metodologia da alocação sequencial ou escalonar utilizando-se do formato de tabelas no banco de dados, sem a utilização da álgebra matricial, simplificando o processo através da utilização dos recursos computacionais. O relato dessa experiência está no artigo de Souza e Scatena (2009).

Ao se questionar sobre as diferenças que poderiam ocorrer nos custos dos centros finais ou produtivos com a utilização da metodologia de alocação escalonar ou sequencial, decidiu-se pela realização dos cálculos da alocação, aplicando-se as metolodologias de alocação direta e alocação sequencial ou escalonar utilizando-se do software Excel da Microsoft.

Assim, tornou-se possível realizar este trabalho e comparar os resultados, podendo-se verificar o impacto da mudança da metolodologia nos valores dos centros de custos finais e dos custos médios unitários.

\section{COMPARAÇÃO DAS METODOLOGIAS}

No processo de apuração de custos por centro de custos, o primeiro passo é a identificação dos custos diretos gastos por cada centro de custos. Assim, no hospital em estudo, esses valores foram levantados através do sistema de informação e também de fontes documentais obtidas junto à administração do município, visto que o hospital não tem autonomia financeira. Em seguida, o item denominado custo indireto geral, que nesse caso se referiu apenas à energia elétrica, foi distribuído a cada centro de custo tendo por critério a quantidade ponderada de equipamentos e demais elementos que consomem energia. $\mathrm{O}$ valor rateado foi de $\mathrm{R} \$ 11.924,57$, de modo que o valor dos centros de custos após o rateio do custo indireto geral totalizou $\mathrm{R} \$ 445.526,47$.

Para aumentar a possibilidade de comparação, decidiu-se, inicialmente, realizar o rateio utilizando-se da metodologia de alocação direta, resultando nos valores dos centros de custos finais e externo demonstrados na tabela 1. Ressalta-se que os centros de custos externos se referem às unidades de saúde que recebem serviços prestados pelo hospital, como por exemplo: exames laboratoriais, exames de raios-x, ultra-sonografia, etc.

Tabela 1 - Total dos centros de custos após alocação direta

\begin{tabular}{c|c|c|c|c|c|c}
\hline Cl. Cirúrgica & Cl. G.O. & Cl. Médica & Cl. Pediátrica & PS/Amb. & Externos & Total \\
\hline $62.852,80$ & $84.095,20$ & $76.168,68$ & $66.370,57$ & $124.723,92$ & $31.315,30$ & $445.526,47$ \\
\hline
\end{tabular}

Em seguida foi realizado o rateio utilizando-se da metolodogia de alocação sequencial ou escalonar. Quanto à ordem dos centros de custos, administrativos e intermediários, Falk (2001) afirma que a ordem pode ser em função da quantidade de centros para os quais o centro administrativo ou intermediário prestou serviços ou em função do valor alocado no centro administrativo ou intermediário. Assim, num primeiro momento a ordem foi realizada levando em consideração apenas o primeiro critério, resultando nos valores demonstrados na tabela 2. Convém destacar que nessa metodologia é possível verificar o valor inicial de cada centro de custo administrativo ou intermediário e também seu valor após o recebimento do rateio do centro que está acima dele na ordem escalonar. No entanto, para os fins deste trabalho limitar-se-à na apresentação dos valores dos centros de custos finais e externos.

Tabela 2 - Total dos centros de custos após alocação sequencial ou escalonar (quantidade)

\begin{tabular}{l|c|c|c|c|c|c}
\hline Cl. Cirúrgica & Cl. G.O. & Cl. Médica & Cl. Pediátrica & PS/Amb. & Externos & Total \\
\hline
\end{tabular}




\begin{tabular}{l|l|l|l|l|l|l}
$64.156,55$ & $88.771,61$ & $73.983,01$ & $63.106,28$ & $122.895,92$ & $32.613,10$ & $445.526,47$ \\
\hline
\end{tabular}

Tendo em mente o segundo critério de ordenação dos centros de custos apresentado por Falk (2001), decidiu-se pela utilização do mesmo como critéiro de desempate, visto que alguns centros administrativos e intermediários apresentavam a mesma quantidade de centros de destino. Assim, em caso de empate, essa se apresenta como uma boa opção de resolução do problema. Com base nesse critério, os valores dos centros de custos finais são demonstrados na tabela 3 .

Tabela 3 - Total dos centros de custos após alocação sequencial ou escalonar (valores)

\begin{tabular}{c|c|c|c|c|c|c}
\hline Cl. Cirúrgica & Cl. G.O. & Cl. Médica & Cl. Pediátrica & PS/Amb. & Externos & Total \\
\hline $64.147,19$ & $88.751,75$ & $73.978,89$ & $63.106,55$ & $122.901,42$ & $32.640,68$ & $445.526,47$ \\
\hline
\end{tabular}

Os valores da tabela 3 não demonstraram diferença significativa entre uma forma de ordenação e outra. No entanto, embora a diferença não seja tão significativa, em caso de empate quanto às quantidades, como ocorreu nesse caso, a utilização dos valores como critério de desempate propicia uma sequencia mais racional e menos arbitrária.

Resta-nos agora trazer a resposta à grande questão levantada neste trabalho: Existe diferença significativa nos valores dos centros finais quando utilizada a metodologia de alocação escalonar ou sequencial ou alocação recíproca? Para dar uma amplitude um pouco maior à comparação, apresenta-se na tabela 4 os valores obtidos a partir da utilização das três metodologias, ou seja, direta, escalonar ou sequencial e recíproca ou matricial. Quanto à alocação escalonar será utilizado nessa comparação os valores demonstrados na tabela 3 . Quanto à alocação recíproca serão utilizados os valores obtidos através da utilização do software SIGHO, conforme comentado na metodologia.

Tabela 4 - Total dos centros de custos após alocação sequencial ou escalonar (valores)

\begin{tabular}{l|c|c|c|c|c|c|c}
\hline Metodologia & Cl. Cirúrg. & Cl. G.O. & Cl. Médica & Cl. Pediát. & PS/Amb. & Externos & Total \\
\hline Direta & $62.852,80$ & $84.095,20$ & $76.168,68$ & $66.370,57$ & $124.723,92$ & $31.315,30$ & $445.526,47$ \\
\hline Escalonar & $64.147,19$ & $88.751,75$ & $73.978,89$ & $63.106,55$ & $122.901,42$ & $32.640,68$ & $445.526,47$ \\
\hline Recíproca & $63.410,28$ & $88.637,06$ & $73.830,31$ & $61.769,64$ & $125.026,44$ & $32.852,60$ & $445.526,33$ \\
\hline Diferença 1 & $\mathbf{5 5 7 , 4 8}$ & $\mathbf{4 . 5 4 1 , 8 6}$ & $\mathbf{- 2 . 3 3 8 , 3 7}$ & $\mathbf{- 4 . 6 0 0 , 9 3}$ & $\mathbf{3 0 2 , 5 2}$ & $\mathbf{1 . 5 3 7 , 3 0}$ & \\
\hline Diferença 2 & $\mathbf{- 7 3 6 , 9 1}$ & $\mathbf{- 1 1 4 , 6 9}$ & $\mathbf{- 1 4 8 , 5 8}$ & $\mathbf{- 1 . 3 3 6 , 9 1}$ & $\mathbf{2 . 1 2 5 , 0 2}$ & $\mathbf{2 1 1 , 9 2}$ & \\
\hline
\end{tabular}

Como é possível notar na tabela 4, o valor total da alocação recíproca sofreu uma pequena redução de $\mathrm{R} \$ 0,14$ centavos quando de sua operacionalização através do software. Esta diferença ocorreu em virtude das muitas interações que o sistema procedeu, oriundas da reciprocidade existente entre os centros de custos.

A comparação dos valores demonstra que no item diferença 1 da tabela 4 a comparação foi entre a alocação recíproca e a alocaçao direta. Nessa comparação, fica evidente que a diferença entre essas duas metodologias é significativa, embora não tenha chegado a 10\%. Como já destacado, a alocação direta não considera a reciprocidade entre os centros, apenas distribui os custos diretamente dos centros administrativos e intermediários aos finais e externos. Assim, corroboramos a afirmação de Martins (1996) de que a alocação recíproca é mais precisa, no entanto, a diferença poderia ter sido maior, caso houvesse uma maior reciprocidade entre os centros administrativos e intermediários.

$\mathrm{O}$ item diferença 2 demonstra a comparação da metodologia de alocação recíproca com a escalonar, no entanto, fica evidente também a diferença em relação à comparação anterior, a qual reduziu significativamente e se alterou quanto ao centro de custos. As diferenças mais significativas estão nas clínicas G. O., médica e pediátrica. 
Quanto à comparação entre a metodologia escalonar e a recíproca, fica evidente que a diferença não é tão significativa, não chegando nem mesmo a $3 \%$, seja para mais ou para menos, no entanto, o modo como esta metodologia é operacionalizada torna os valores realmente mais precisos.

Segundo Martins (1996), a metodologia de alocação escalonar ou sequencial é a mais utilizada em função da facilidade de ser operacionalizada, no entanto, como se percebe no exemplo, não é a mais precisa. No caso do centro de custo PS/Amb com alocação sequencial ou escalor o valor do custo total estaria subavaliado em $\mathrm{R} \$ 2.125,02$, enquanto a clínica pediátrica teria seus valores superavaliados em $\mathrm{R} \$ 1.336,91$.

\title{
5 CONSIDERAÇÕES FINAIS
}

Os resultados oriundos da comparação entre as metodologias ratifica a afirmação do Ministério da Saúde (2006) e de Falk (2001) de que a metologia de alocação recíproca, embora mais complexa de ser operacionalizada, apresenta resultados mais precisos e melhores.

É verdade que os valores apresentados não evidenciaram grande expressividade na diferença entre a metodologia sequencial ou escalonar e a recíproca. Embora a diferença não seja tão expressiva ela existe e a metodologia de alocação recíproca é realmente mais precisa, em função do modo mais racional como é operacionalizada.

É importante destacar que a diferença entre as metodologias sofre influência direta do volume de reprocidades existentes, ou seja, se houvesse ocorrido maior número de prestação de serviços dos centros intermediários aos administrativos e vice-versa, com certeza a diferença seria bem mais significativa.

Ressalta-se que através da utilização de um software específico para a operacionalização do processo, é possível realizar o cálculo utilizando a metodologia de alocação recíproca com bastante sucesso, conforme demonstra a experiência de Souza e Scatena (2009). Através de um software é possível, inclusive, ter no desenvolver aplicativos que utilizem a mesma base de dados para calcular os custos com base nas três metodologias e gerar relatórios para comparação.

Assim, o contador, ao realizar o cálculo dos custos de produção, deve prezar pela maior racionalidade do processo e exatidão dos valores apresentados, de modo que as distorções nos valores sejam reduzidas ao mínimo possível. Diante desse desafio, a metodologia de alocação recíproca se mostra numa ferramenta muito útil e que auxilia no cumprimento dessa missão por parte dos contadores, ou seja, apurar custos com o objetividade e racionalidade, máximizando a exatidão e mínimizando as arbitrariedades e distorções.

\section{REFERÊNCIAS}

\begin{abstract}
ARAÚJO, R.G.; NASCIMENTO D.T. Utilização do modelo de alocação recíproca de custos para a verificação do nível de imprecisão no rateio de custos indiretos de uma indústria farmacêutica: estudo de caso. [artigo na internet]. São Paulo; 2007 [acesso em 22 de fevereiro de 2009]. Disponível em: www.congressoeac.locaweb.com.br/artigos72007/213.pdf
\end{abstract}

BEULKE, R.; BERTÓ, J.D. Gestão de custos e resultados na saúde: hospitais, clínicas, laboratórios e congêneres. 4. ed. rev. atualizada e ampliada. São Paulo: Saraiva, 2008.

BRUNI, A.L.; FAMÁ, R. Gestão de custos e formação de preços: com aplicações na calculadora HP 12C e Excel. 3 . ed. São Paulo: Atlas, 2004.

CHING, Y.H. Manual de custos de instituições de saúde: sistemas tradicionais de custos e 
sistema de custeio baseado em atividades (ABC). São Paulo: Atlas, 2001.

FALK, J.A. Tecnologia de informação para gestão de custos e resultados no hospital: considerações e dicas para implantação de um modelo brasileiro. São Paulo: Atlas, 2008.

FALK, J.A. Gestão de custos para hospitais: conceitos, metodologias e aplicações. São Paulo: Atlas, 2001.

HRQ Consultoria. Alocação de Custos. [200-]. Disponível em: $<$ http://www.hrqconsultoria.com.br/index.php/joomla-overview/alocacao-de-custos>. Acesso em: 20 maio 2010. Não paginado.

MARTINS, E. Contabilidade de custos. 5a . ed. rev. São Paulo: Atlas, 1996.

MARTINS, G.A. Métodos convencionais e não-convencionais e a pesquisa em administração. Caderno de pesquisas em administração. V. 00, No. 0, 2 . Semestre, 2004.

MINISTÉRIO DA SAÚDE. Secretaria de Atenção à Saúde. DATASUS. Cadastro Nacional de Estabelecimentos de Saúde - CNES. Brasília; 2008. [acesso dia 22 de fevereiro de 2008]. Disponível em: http://cnes.datasus.gov.br/Lista_Es_Nome.asp?VTipo=0

MINISTÉRIO DA SAÚDE. Secretaria de Ciência, Tecnologia e Insumos Estratégicos. Departamento de Economia da Saúde. Programa Nacional de Gestão de Custos. Manual Técnico de Custos: conceitos e metodologia. Brasília, DF; 2006.

PADOVEZE, C. L. Curso básico gerencial de custos. $2^{\mathrm{a}}$. ed. rev. e ampl. São Paulo: Pioneira Thompson Learning, 2006.

SCARDOELLI, L. Y. ; NAGANO, M. S. ; PEREIRA, E. Desenvolvimento de Sistema Informatizado para a Locação Recíproca de Custos Utilizando Cálculo Matricial. In: X CONGRESSO BRASILEIRO DE CUSTOS, 2003, Guarapari - ES. Anais do X Congresso Brasileiro de Custos, 2003., 2003. v. 1. p. 1-15.

SOUZA, P. C. ; SCATENA, J. H. G. Alocação recíproca de custos hospitalares indiretos sem a aplicação da álgebra matricial. Revista de Contabilidade - UFBA. Salvador, v. 3, n. 1, p. 58-71, janeiro/abril 2009.

SOUZA, P. C. Avaliação econômica em saúde: um estudo de caso do Hospital Municipal de Barra do Bugres-MT. 2008. 181 f. dissertação (Mestrado) - Programa de Pós-Graduação em Saúde Coletiva, Universidade Federal de Mato Grosso, Cuiabá, 2009. 